\title{
TRANSESOPHAGEAL BIOPSY OF MEDIASTINAL AND PULMONARY TUMORS BY MEANS OF ENDOSCOPIC ULTRASOUND GUIDANCE
}

Michael Hünerbein, MD

Bijan Michael Ghadimi, MD

Wolfgang Haensch, MD

Peter M. Schlag, MD, PhD, FSSO
Objective: The aim of this study was to investigate the value of endoscopic ultrasound-guided biopsy for the diagnosis of thoracic lesions. Methods: Transesophageal ultrasound-guided biopsy was performed in 29 patients with mediastinal $(n=25)$ or pulmonary tumors $(n=4)$. A flexible echoendoscope with a 7.5 MHz curved array transducer (Pentax FG 32 UA, Hamburg, Germany) and a biopsy device with a fine needle (diameter $0.8 \mathrm{~mm}$ ) were used for all examinations. Three patients were excluded from the analysis of the data because a definite diagnosis based on surgery or follow-up was not available. Results: Real-time visualization of the biopsy procedure with endoscopic ultrasound enabled accurate tissue sampling even of small mediastinal lesions with a diameter of less than $1 \mathrm{~cm}$. Diagnostic material was obtained in 23 of the 26 patients $(88 \%)$. In 3 cases $(12 \%)$ non-representative biopsy material was found in the specimen. The sensitivity and specificity of transesophageal biopsy in the diagnosis of malignancy were $89 \%$ and $83 \%$, respectively. Histologic analysis of the biopsy specimens established malignancy in 17 of 23 patients, whereas benign lesions were diagnosed in 6 patients. Endoscopic ultrasound-guided biopsy confirmed the diagnosis suggested by conventional diagnostic methods in 15 of 23 patients $(65 \%)$, whereas an unsuspected diagnosis was disclosed in 8 patients $(35 \%)$. The results of the biopsy had considerable impact on the therapeutic strategy. None of the patients had complications related to the procedure. Conclusions: Endoscopic ultrasound-guided biopsy provides a new minimally invasive approach to the biopsy of lesions in the posterior mediastinum and may complement surgical staging procedures. (J Thorac Cardiovasc Surg 1998;116:554-9)
$T^{\text {he }}$ he value of computed tomography and magnetic resonance imaging for imaging of mediastinal tumors and lymph nodes has been evaluated extensively. ${ }^{1,2}$ Although mediastinal lesions can be visualized with computed tomography and magnetic resonance imaging, a definitive diagnosis is generally not obtained solely on the basis of morphologic characteristics. ${ }^{3}$

In recent years endoscopic ultrasound (EUS) has been increasingly used for staging of esophageal cancer. This

From the Charitè University Hospital, Robert Rössle Hospital and Tumor Institute, Humboldt University, Berlin, Germany.

Received for publication Dec 19, 1997; revisions requested March 17, 1998; revisions received April 23, 1998; accepted for publication April 24, 1998.

Address for reprints: P. M. Schlag, MD, Department of Surgery and Surgical Oncology, Robert-Rössle Hospital and Tumor Institute, Humboldt University, Lindenbergerweg 80, 13122 Berlin, Germany.

Copyright $(\odot) 1998$ by Mosby, Inc.

0022-5223/98 $\$ 5.00+0 \quad \mathbf{1 2 / 1 / 9 1 3 7 0}$ technique provides high-resolution images of the intestinal wall and the paraesophageal region. Generally the sensitivity of EUS in the detection of mediastinal lymph node involvement approximates $80 \%$, and there is some evidence that EUS is more accurate than computed tomography (69\% vs $51 \%) .{ }^{4}$ However, it remains difficult to distinguish malignant and benign lesions reliably. The specificity of EUS in the identification of metastatic lymph node involvement ranges between $50 \%$ and $70 \% .{ }^{5}$ Therefore mediastinal biopsy is still necessary to achieve a precise diagnosis of mediastinal lesions. ${ }^{6}$

Recently echoendoscopes with linear-array transducers have been developed that allow EUS-guided biopsy of extraluminal lesions. ${ }^{7}$ However, as yet mainly preliminary information is available on the relevance of this new method for the evaluation of mediastinal tumors. ${ }^{7-9}$ The purpose of this prospective study was to investigate the value of EUS-guided biopsy for the diagnosis of mediastinal lesions. 

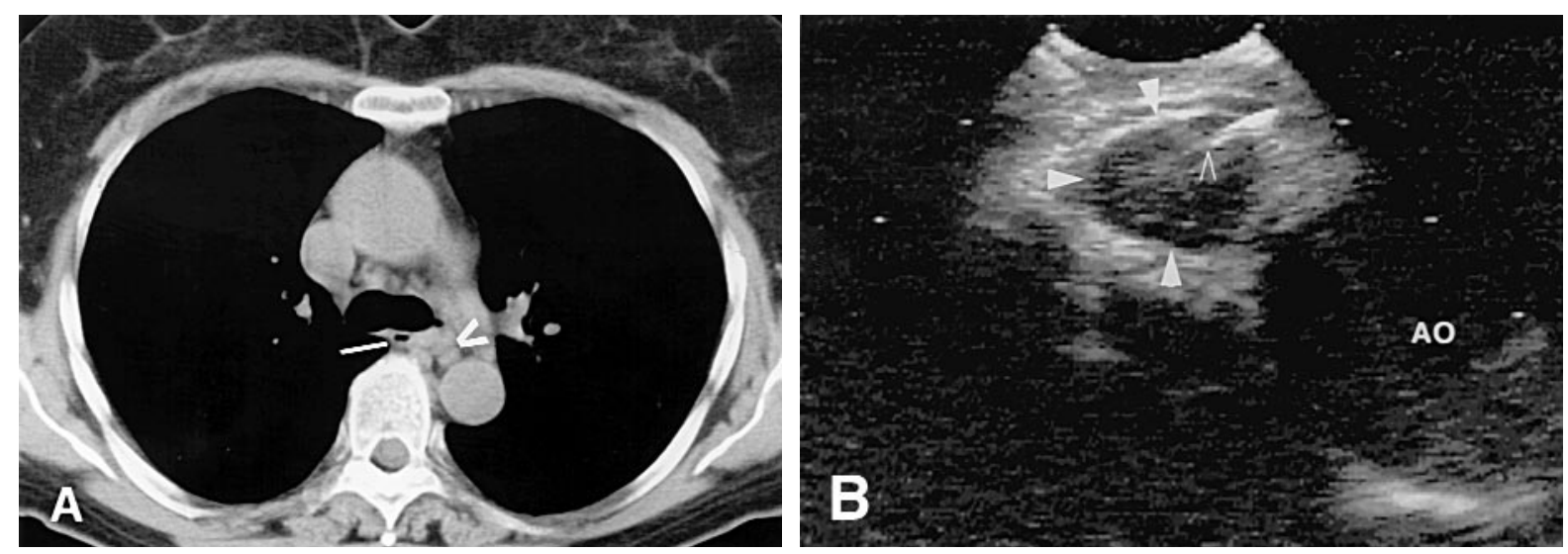

Fig 1. A, Computed tomographic scan shows mediastinal lymph nodes (arrow) between esophagus (-) and aorta. B, EUS accurately displays the needle (arrow) in a small para-aortic lymph node (arrowheads). AO, Aorta.

\section{Patients and methods}

Overall, 81 patients underwent EUS with ultrasound-guided fine needle biopsy for the evaluation of mediastinal $(\mathrm{n}=$ 29) or abdominal tumors $(n=52)$. Informed consent was obtained from all patients. Transesophageal biopsy was performed in 25 patients with mediastinal lesions and 4 patients with pulmonary tumors. The patients comprised 17 men and 12 women with a mean age of 58 years (range, 38-74 years). Three patients with mediastinal lesions were excluded from the analysis of the data because the diagnostic information was not yet sufficient to establish a precise diagnosis.

For EUS the patients were placed in the left lateral decubitus position. Midazolam (3-5 mg) was administered intravenously for conscious sedation. A 60-degree oblique viewing fiberoptic endoscope (Pentax FG 32UA, Pentax, Hamburg, Germany) with a curved array transducer (7.5 $\mathrm{MHz}$ ) was used for all examinations. The 100-degree scan plane of the transducer is oriented along the longitudinal axis of the endoscope, which allows real-time visualization of the biopsy needle. EUS images were displayed on a CS9600 ultrasound unit (Ecoscan, Wiesbaden, Germany).

A specially designed needle device (Mandel \& Rupp, Erkrath, Germany) was used for EUS-guided aspiration biopsy. ${ }^{10}$ The device consists of a $140-\mathrm{cm}$ long stainless steel needle with a diameter of $0.8 \mathrm{~mm}$, which is guided through a $5 \mathrm{~F}$ metal sheath. The biopsy needle can be advanced with a handle. When a lesion was displayed with EUS, catheter and needle were passed through the working channel until the catheter just reached the viewing field. Then the needle was filled with sterile saline solution and introduced into the target. Fine needle aspiration biopsy was achieved by applying suction with a 5-ml syringe while the needle was advanced and withdrawn several times. Generally 4 to 6 passes were required to obtain adequate tissue samples. The duration of the whole procedure ranged between 10 and 20 minutes.

The biopsy specimens were placed in formaldehyde. Conventional staining was used for histologic analysis.
Additionally, immunohistochemistry was performed in selected cases if the results of routine staining were inconclusive.

All patients underwent a follow-up program with examinations every 3 months. Progressive disease confirmed malignancy in all patients whose biopsy specimens had histologic characteristics of malignant disease. Surgery was performed in 4 of 6 patients with benign histologic characteristics and confirmed the diagnosis in all cases. In the 2 remaining patients with a history of cancer (esophageal cancer and nonHodgkin's lymphoma) and benign histologic characteristics, there is no evidence of malignancy after follow-up periods of 9 and 7 months, respectively.

\section{Results}

EUS with the 7.5-MHz curved array transducer provided high-resolution images of the gastrointestinal tract and the adjacent structures. The longitudinal scan plane of the transducer enabled accurate real-time visualization of the needle during the biopsy procedure (Figs 1 and 2). This technique allowed the precise biopsy of even small mediastinal lesions with a diameter of less than $1 \mathrm{~cm}$. Diagnostic tissue samples were obtained in 23 of 26 patients $(88 \%)$. All specimens were adequate for histopathologic assessment. Additionally, immunohistochemistry was performed in 7 cases to achieve a definite classification of the tumor (Fig 2,C). On the basis of histologic study, malignancy was diagnosed in 17 of 23 patients $(74 \%)$ whereas benign lesions were found in 6 patients (26\%). Transesophageal biopsy failed to establish a correct diagnosis in 3 of 26 patients (12\%). Recurrent esophageal cancer was observed in 1 patient although transesophageal biopsy had shown a benign lymph node a few months before. In 2 cases fine needle aspiration of a paraesophageal lymph node yielded nondiagnostic material (ie, fibrotic and fatty tis- 

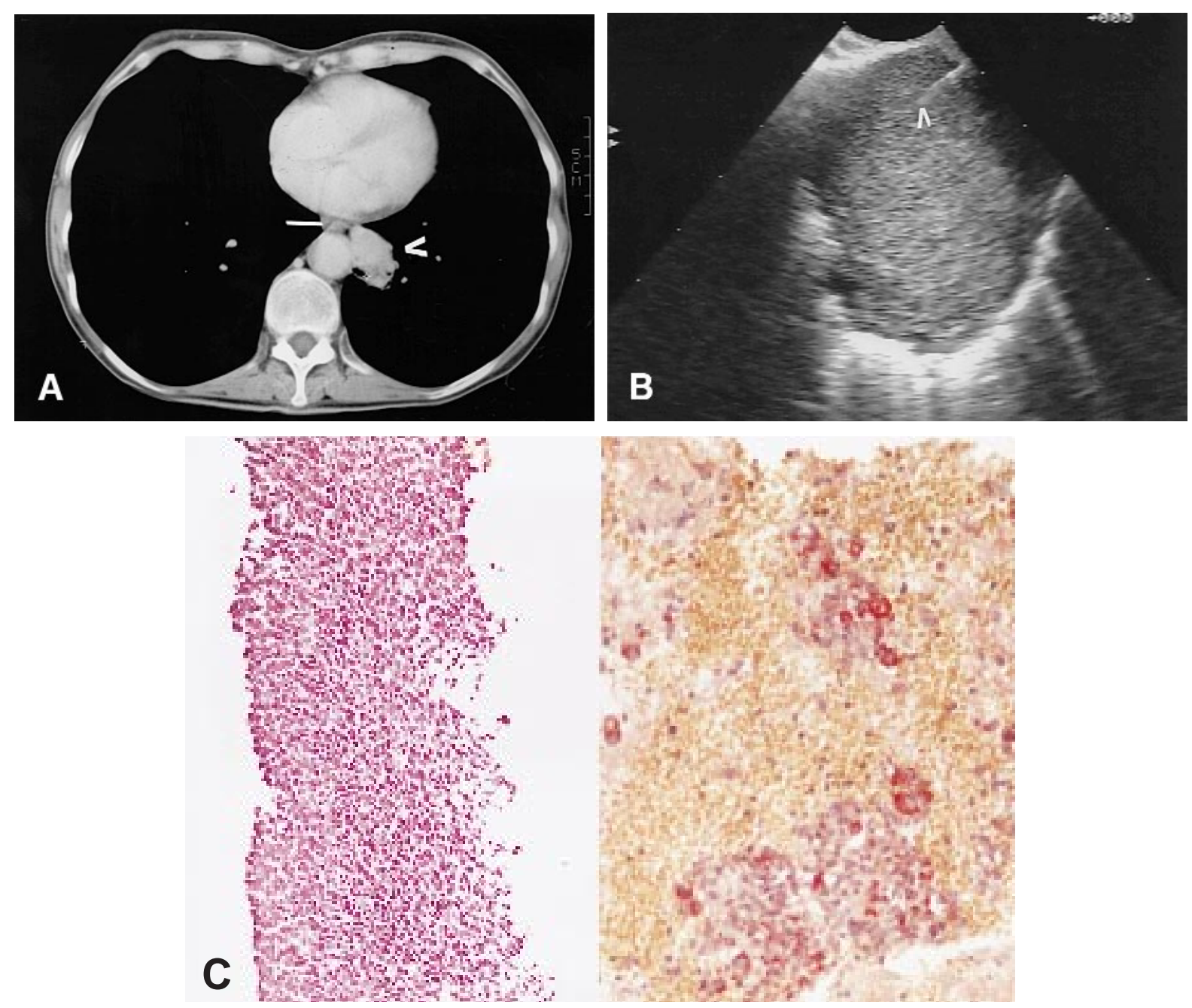

Fig 2. A, Computed tomography demonstrates a pulmonary tumor (arrow) at the left hilum. -, Esophagus. B, Transesophageal EUS-guided biopsy of the lesion with a fine needle (arrow). C, Core biopsy and immunohistochemistry with neuron-specific enolase-positive reddish cells demonstrate a small cell carcinoma.

sue). The detailed results of EUS-guided biopsy are shown in Table I.

EUS-guided biopsy improved the assessment of mediastinal lymph nodes (Table II) and had considerable impact on the diagnosis and therapy of mediastinal tumors. Biopsy confirmed the suspected diagnosis and provided a precise histologic classification of the tumor in 15 of 23 patients (65\%). In 8 patients (35\%) histologic study altered the diagnosis suggested by the conventional diagnostic evaluation (Table III). In 2 patients with suspected recurrent esophageal cancer and non-Hodgkin's lymphoma, histologic examination revealed an inflammatory lymph node and sarcoidosis, respectively. The first patient remains without evidence of recurrence after a follow-up of 7 months, whereas sarcoidosis was confirmed by mediastinoscopy. In 2 further patients with "mediastinal non-Hodgkin's lymphoma," fine needle aspiration disclosed cancer of an unknown primary (adenocarcinoma) and small cell carcinoma of the lung. Although imaging suggested cancer of the lung, recurrent gastric cancer was diagnosed in another patient 10 years after resection of the primary tumor.

Mediastinal metastases of extrathoracic primary tumors were excluded in 2 patients by EUS-guided biopsy. Histologic examination revealed a mediastinal goiter in a patient with a malignant melanoma of the neck, a diagnosis that was subsequently also established by scintigraphy. A secondary tumor, a small cell carcinoma of the lung, was found in a patient who had undergone nephrectomy for a renal carcinoma.

The overall sensitivity and specificity of EUS-guided biopsy for the diagnosis of malignancy were $89 \%$ and 
Table I. Histopathologic diagnosis of EUS-guided biopsy in 29 patients with mediastinal and pulmonary lesions

\begin{tabular}{llc}
\hline Location & \multicolumn{1}{c}{ Histology } & No. \\
\hline $\begin{array}{l}\text { Mediastinal lymph nodes } \\
(\mathrm{n}=19)\end{array}$ & Recurrent gastric cancer & 3 \\
& Lymphoma & 1 \\
& Adenocarcinoma & 1 \\
& Small cell carcinoma & 1 \\
& Prostate cancer & 1 \\
& Recurrent esophageal cancer & 4 \\
& Benign lymph node & 5 \\
Mediastinal mass lesions & Non-representative & 3 \\
$(\mathrm{n}=6)$ & Sarcoma & 2 \\
& Melanoma & 1 \\
& Thymoma & 1 \\
& Small cell carcinoma & 1 \\
Pulmonary tumors & Goiter & 1 \\
$(\mathrm{n}=4)$ & Small cell carcinoma & 3 \\
& Adenocarcinoma & 1
\end{tabular}

$83 \%$, respectively. The positive predictive value was $100 \%$ and the negative predictive value was $75 \%$. The procedure was well tolerated by the patients and no complications were observed related to transesophageal biopsy.

\section{Discussion}

Because of the morbidity associated with diagnostic thoracotomy, less-invasive procedures such as mediastinoscopy have been developed for tissue sampling of mediastinal lesions. ${ }^{11-13}$ In recent years thoracoscopy has been increasingly used for evaluation of mediastinal lesions that are inaccessible to the mediastinoscope. The morbidity rate of both procedures is low. ${ }^{14}$ However, albeit rare, major complications such as hemorrhage and recurrent nerve palsy have been observed. ${ }^{15,16}$ Furthermore, general anesthesia and hospitalization are usually required for both techniques.

The development of echoendoscopes with curved array transducers offers a new modality for ultrasoundguided biopsy of mediastinal lesions. However, as yet only limited experience has been gained with this method, mainly because of the lack of appropriate biopsy needles.

In our experience, EUS with fine needle aspiration proved to be a very safe and accurate method for tissue sampling of mediastinal lesions. This technique provides access to regions that are not easily accessible by cervical mediastinoscopy, that is, the posterior and inferior mediastinum. However, visualization of lesions in the anterior mediastinum is not possible because the airfilled trachea prevents ultrasound transmission.

Real-time ultrasound visualization of the biopsy pro-
Table II. Correlation of endosonographic diagnosis and histology of EUS-guided biopsy in 16 patients with mediastinal lymph nodes

\begin{tabular}{lcc}
\hline & \multicolumn{2}{c}{ Histology } \\
\cline { 2 - 3 } EUS diagnosis & Malignant & Benign \\
\hline Malignant lymph node & 10 & 3 \\
Benign lymph node & 1 & 2 \\
\hline
\end{tabular}

Table III. EUS-guided biopsy changed the diagnosis in 8 of 23 patients

\begin{tabular}{|c|c|c|}
\hline Suspected diagnosis & Histology & $\begin{array}{l}\text { Therapeutic } \\
\text { consequence }\end{array}$ \\
\hline \multicolumn{3}{|l|}{ Mediastinal primary } \\
\hline \multirow[t]{3}{*}{ Lymphoma $(\mathrm{n}=3)$} & Adenocarcinoma (CUP) & Chemotherapy \\
\hline & Small cell carcinoma & Chemotherapy \\
\hline & Sarcoidosis* & Medical therapy \\
\hline $\begin{array}{l}\text { Recurrent esophageal } \\
\text { cancer }\end{array}$ & Benign lymph node $\dagger$ & Follow-up \\
\hline Lung cancer & Recurrent gastric cancer & Chemotherapy \\
\hline \multicolumn{3}{|l|}{ Mediastinal secondary } \\
\hline Malignant melanoma & Goiter & No therapy \\
\hline Renal cell carcinoma & Small cell carcinoma & Chemotherapy \\
\hline Sarcoma & Complete necrosis & Resection \\
\hline
\end{tabular}

$C U P$, Cancer of unknown primary.

*Confirmation by mediastinoscopy.

$\nmid$ Confirmation by 7-month follow-up.

\$Confirmation by scintigraphy.

cedure enabled highly precise biopsy of small lesions even in direct proximity to major vessels. Overall, diagnostic biopsy specimens were obtained in $88 \%$ of the cases (sensitivity, $89 \%$; specificity, $83 \%$ ). The high accuracy of EUS-guided biopsy of mediastinal tumors compares favorably with the results reported for gastrointestinal tumors. In a group of 45 patients with pancreatic tumors, Faigel and associates ${ }^{17}$ reached a rate of 91\% diagnostic fine needle aspirations under EUS guidance. Giovanini and coworkers ${ }^{18}$ achieved a sensitivity of $77 \%$ and a specificity of $100 \%$ in the detection of malignancy in 141 patients with various tumors. Recently, encouraging results of EUS-guided biopsy of mediastinal tumors have been presented in a preliminary report. ${ }^{19}$ A conclusive cytologic study was obtained and confirmed in 12 of 13 lesions. In contrast to this and some other studies, ${ }^{7,20}$ in our study the diagnosis was based on histologic analysis and not on cytologic examination. The size and the quality of the tissue samples were sufficient for routine staining and histopathologic assessment of the biopsy specimen. It is remarkable that immunohistochemistry could also be performed in some cases to specify the diagnosis. 
Ultrasound-guided biopsy greatly increased the accuracy of EUS in the classification of mediastinal lymph nodes (ie, malignant vs benign). Histologic study disclosed a false positive EUS diagnosis in 3 of 13 patients with suspected malignant lymph nodes. On the other hand, metastatic spread was found in 1 of 3 patients although benign lymph nodes were suggested by EUS. These data demonstrate that EUS-guided biopsy is capable of improving the staging of mediastinal lymph nodes, which can be of major importance for the therapeutic strategy in patients with thoracic tumors.

Recently, Silvestri and associates ${ }^{21}$ evaluated the utility of this modality for staging of lung cancer in 27 patients. EUS-guided fine needle aspiration biopsy enabled detection of lymph node metastases with a sensitivity of $89 \%$. It has also been reported that this technique is more accurate in the assessment of lymph node involvement from lung cancer than computed tomography $(95 \%$ vs $43 \%) .{ }^{22}$ Surgery was abandoned in $59 \%$ of the patients, because mediastinal lymph node metastases were established by transesophageal biopsy.

Besides improved staging of known primary tumors, EUS-guided biopsy can provide a histopathologic diagnosis of indeterminate mediastinal tumors that are not accessible for other nonsurgical techniques, for example, bronchoscopy. In this study biopsy confirmed the suspected diagnosis in 19 of 27 patients, whereas an unexpected diagnosis was disclosed in 8 patients $(30 \%)$. Notably, biopsy did not confirm metastases in 2 patients with suspicious mediastinal lymph nodes and a history of cancer. In a patient with a melanoma, biopsy showed an intrathoracic goiter, whereas a secondary tumor (lymphoma) was found in a patient with a hypernephroma. These findings support the need for histologic confirmation of the diagnosis even in apparently clear cases.

We have not observed any complications related to EUS-guided biopsy. However, needle tract seeding can occur after transcutaneous needle biopsy of malignant tumors. In a follow-up survey involving 95,000 fine needle biopsies, metastases in the biopsy canal were observed in only 6 cases $(0.006 \%) .{ }^{23}$ Overall, the risk of tumor cell dissemination by fine needle biopsy seems to be low. Nonetheless, we would suggest that EUS-guided biopsy be preferentially applied to patients with nonresectable or metastatic disease.

In conclusion, EUS with ultrasound-guided biopsy is a valuable method for the diagnostic evaluation of mediastinal tumors and the staging of lymph nodes. This minimally invasive technique allows precise tissue sampling of lesions in the posterior mediastinum. In selected cases, EUS-guided biopsy can obviate the need for diagnostic surgical procedures, that is, mediastinoscopy and thoracoscopy.

\section{REFERENCES}

1. Bollen EC, Goei R, v.'t.Hof-grootenboer BE, Versteege CWM, Engelshove HA, Lamers RJ. Interobserver variability and accuracy of computed tomographic assessment of nodal status in lung cancer. Ann Thorac Surg 1994;58:158-62.

2. Dillemanns B, Deneffe G, Verschakelen J, Decramer M. Value of computed tomography and mediastinoscopy in preoperative evaluation of mediastinal lymph nodes in non small cell lung cancer. Eur J Cardiothorac Surg 1994;8:37-42.

3. Pugatch RD. Radiologic evaluation in chest malignancies. Chest 1995;107(suppl):294-7.

4. Ziegler K, Sanft C, Zimmer T, Zeitz M, Felsenberg D, Stein H, et al. Comparison of computed tomography, endosonography, and intraoperative assessment in TN staging of gastric carcinoma. Gut 1993;34:604-10.

5. Roesch T. Endoscopic ultrasonography. Endoscopy 1994;26:14868.

6. Fry WA. Biopsy of lesions of the thorax. Surg Oncol Clin North Am 1995;4:29-46.

7. Vilmann P, Hancke S, Henriksen FW, Jacobsen GK. Endosonographically guided fine needle aspiration biopsy of malignant lesions in the upper gastrointestinal tract. Endoscopy 1993; 25:523-7.

8. Hünerbein M, Dohmoto M, Rau B, Schlag PM. Endosonography and endosonography guided biopsy of upper GI-tract tumors using a curved array echoendoscope. Surg Endosc 1996;10:1205-9.

9. Wegener M, Adamek RJ, Wedmann B, Pfaffenbach B. Endosonographically guided fine needle aspiration puncture of paraesophagogastric mass lesions: preliminary results. Endoscopy 1994;26:586-91

10. Vilmann P, Hancke S. A new biopsy handle instrument for endoscopic ultrasound guided fine needle aspiration biopsy. Gastointest Endosc 1996;43:238-42.

11. Rendina EA, Venuta F, DeGiacomo T, Ciriaco PP, Pescarmona EO, Francioni F, et al. Comparative merits of thoracoscopy, mediastinoscopy and mediastinotomy for mediastinal biopsy. Ann Thorac Surg 1994;57:992-5.

12. Goldstraw P. The role of mediastinoscopic biopsy in preoperative assessment of lung cancer. J Thorac Cardiovasc Surg 1994;107: 309-11.

13. Yim AP. Minimizing chest wall trauma in video-assisted thoracic surgery. J Thorac Cardiovasc Surg 1995;109:1255-6.

14. Luke WP. Prospective evaluation of mediastinoscopy for assessment of carcinoma of the lung. J Thorac Cardiovasc Surg 1986;91:553-7.

15. Pearson FG. Staging of the mediastinum. Chest 1993;103:346-8.

16. Ricci C, Rendina EA, Venuta F, Pescarmona EO, Di Tolla R, Ruco LP, et al. Surgical approach to isolated mediastinal lymphoma. J Thorac Cardiovasc Surg 1990;99:691-5.

17. Faigel DO, Ginsberg GG, Bentz JS, Gupta PK, Smith DB, Kochmann ML. Endoscopic ultrasound-guided real time fine needle aspiration biopsy of the pancreas in cancer patients with pancreatic lesions. J Clin Oncol 1997;15:1439-43.

18. Giovanini M, Seitz JF, Monges G, Perrier H, Rabbia I. Fine-needle aspiration cytology guided by endoscopic ultrasonography: results in 141 patients. Endoscopy 1995;27:171-7.

19. Pedersen BH, Vilmann P, Folke K, Jacobsen GK, Krasnik M, Milman N, et al. Endoscopic ultrasonography and real time guided fine needle aspiration biopsy of solid lesions of the mediastinum suspected of malignancy. Chest 1996;110:539-44.

20. Binmoeller KF, Seifert H, Soehendra N. Endoscopic ultrasonog- 
raphy-guided fine-needle aspiration biopsy of lymph nodes. Endoscopy 1994;26:780-3.

21. Silvestri GA, Hoffman BJ, Buthani MS, Hawes RH, Coppage L, Sanders-Cliette A, et al. Endoscopic ultrasound with fine needle aspiration in the diagnosis and staging of lung cancer. Ann Thorac Surg 1996;61:1441-6.

22. Gress FG, Savides TJ, Kesler K. A prospective comparison study of endoscopic ultrasound, computed tomography and EUS directed to fine needle aspiration biopsy of the mediastinum in the preoperative evaluation of small cell lung cancer [abstract]. Gastrointest Endosc 1995;41:304.

23. Weiss H, Duntsch U. Complications of fine needle puncture. DEGUM survey II. Ultraschall Med 1996;17:118-30.

\section{Commentary}

The article by Hünerbein and associates describes the potential usefulness of endoscopic ultrasonography (EUS) with needle biopsy for the evaluation of mediastinal structures adjacent to the esophagus. Their experience mirrors my experience at the Medical University of South Carolina (MUSC). Although EUS is not yet a common procedure in many centers in the United States, I anticipate that its utility will increase as physicians recognize the clinical relevance and very minimally invasive nature of the procedure. EUS with biopsy does require gastroenterologists trained in the technique and close cooperation with the thoracic surgeons.

At MUSC, my colleagues and I have used EUS and fine needle aspiration (FNA) to evaluate more than 140 patients with a lung mass and mediastinal adenopathy. The radi- ographically designated abnormal lymph nodes in both the aortopulmonary window and the subcarinal region are now preferentially evaluated by EUS/FNA. In the period from June 1994 to September 1997, 82 patients with a lung mass and mediastinal adenopathy underwent EUS/FNA. The sensitivity for the diagnosis of malignancy was $88 \%$ and the specificity was $100 \%$. More than 30 patients with abnormal mediastinal adenopathy without a dominant pulmonary mass have also been evaluated by EUS/FNA. Similar to the findings of Hünerbein and colleagues, a variety of disease processes have been diagnosed, including sarcoidosis, histoplasmosis, lymphoma, and small cell lung cancer.

Our experience has been confined to aspiration cytology. The development of a specialized needle that is capable of giving a core biopsy for histologic evaluation may be particularly helpful in cases of lymphoma and granulomatous disease. Like the authors, we have observed no complications from EUS/FNA.

As a diagnostic and staging tool, EUS/FNA is meant to be a complementary procedure to cervical mediastinoscopy and thoracoscopy. In our hands, EUS/FNA is less invasive and more cost effective when applied to the correct situation. In my opinion, other reports will soon verify the results in the present article as surgeons become familiar with the potential usefulness of EUS-guided needle biopsy.

Carolyn E. Reed, MD

Charleston, $S C$ 12/1/91982 